\title{
ARTICLE \\ Intact responses to non-drug rewards in long-term opioid maintenance treatment
}

\author{
Marie Eikemo $\mathbb{D}^{1,2,3}$, Philipp P. Lobmaier ${ }^{1,2}$, Mads L. Pedersen ${ }^{4,5}$, Nikolaj Kunøe ${ }^{1}$, Anna Maria Matziorinis ${ }^{4}$, \\ Siri Leknes ${ }^{4,5}$ and Monica Sarfi ${ }^{1}$
}

\begin{abstract}
Disruption of non-drug reward processing in addiction could stem from long-term drug use, addiction-related psychosocial stress, or a combination of these. It remains unclear whether long-term opioid maintenance treatment (OMT) disrupts reward processing. Here, we measured subjective and objective reward responsiveness in 26 previously heroin-addicted mothers in $>7$ years stable OMT with minimal psychosocial stress and illicit drug use. The comparison group was 30 healthy age-matched mothers (COMP). Objective reward responsiveness was assessed in a two-alternative forced-choice task with skewed rewards. Results were also compared to performance from an additional 968 healthy volunteers (meta-analytic approach). We further compared subprocesses of reward-based decisions across groups using computational modelling with a Bayesian drift diffusion model of decision making. Self-reported responsiveness to non-drug rewards was high for both groups (means: $\mathrm{OMT}=6.59, \mathrm{COMP}=6.67, p=0.84, \mathrm{BF}_{10}=$ 0.29), yielding moderate evidence against subjective anhedonia in this OMT group. Importantly, the mothers in OMT also displayed robust reward responsiveness in the behavioral task $\left(t_{19}=2.72, p=0.013, \mathrm{BF}_{10}=3.98 ; d=0.61\right)$. Monetary reward changed their task behavior to the same extent as the local comparison group (reward bias OMT $=0.12, \mathrm{COMP}=0.12, p=0.96, \mathrm{BF}_{10}=0.18$ ) and in line with data from 968 healthy controls previously tested. Computational modelling revealed that long-term OMT did not even change decision subprocesses underpinning reward behavior. We conclude that reduced sensitivity to rewards and anhedonia are not necessary consequences of prolonged opioid use.
\end{abstract}

Neuropsychopharmacology (2019) 44:1456-1463; https://doi.org/10.1038/s41386-019-0377-9

\section{INTRODUCTION}

Disrupted reward processing is a central component in neurobiological theories of drug addiction [1-5]. In humans, addiction is characterized by exaggerated responses to drugs and drug cues at the expense of pleasure from and motivation for non-drug rewards [6]. Indeed, decreased neural and subjective responses to natural rewards [anhedonia; [7-10]] may predict relapse to drug use [7]. Symptoms of anhedonia have been observed across a range of substance use disorders [11-13], as well as during abstinence [14, 15], withdrawal [16], and pharmacotreatment $[7,15,17]$. Opioid addiction can be considered the prototypical addiction [18], with devastating consequences for individuals and society [19]. The current 'gold standard' treatment is opioid maintenance treatment (OMT) with methadone or buprenorphine [20]. Akin to heroin, OMT medications act as agonists on the opioid receptors, but with long-acting pharmacological properties. OMT reduces illicit opioid use, drug craving, and behavior associated with illegal drug use [20-22]. Patients in stable OMT are relieved of the highs and lows of the stressful 'drug taking cycle', i.e., binge, withdrawal, and drug seeking [1, 23]. However, because these pharmacotreatments act as surrogates at the receptor level, the patients remain opioid dependent in the 'physiological' sense; that is, they are opioid tolerant and will experience withdrawal symptoms if treatment is discontinued. It is unclear whether OMT medications prolong or even cause reward dysregulation. An alternative hypothesis is that patients in longterm stable OMT could exhibit intact reward processing. Life-style related factors such as psychosocial and somatic stress, social stigma, and poly-drug use [24] also contribute to anhedonia.

To determine whether reduced responsiveness to non-drug rewards are a necessary consequence of opioid dependence, we assessed reward responses in a unique group of patients with opioid dependence. Patients had been in stable OMT for $>7$ years, exhibited few psychosocial vulnerability factors compared to the general OMT population, and had minimal concurrent drug use. All patients were mothers who started OMT during pregnancy and who retained custody of their children.

We compared reward responsiveness in OMT patients to a healthy comparison group (COMP) using both subjective (selfreported hedonia) and objective (behavioral) reward responsiveness measures. As a behavioral measure, we used a well-established probabilistic reward task (PRT), which is sensitive to disrupted reward responsiveness during depression [25, 26], stress [27], cannabis dependence, [28] and nicotine withdrawal [29]. Data from the task were analyzed using classical signal detection method as well as with the drift diffusion model of decision making [DDM; [30]]

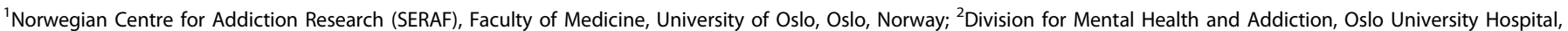

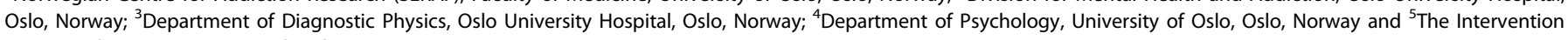
Centre, Oslo University Hospital, Oslo, Norway

Correspondence: Marie Eikemo (m.h.eikemo@medisin.uio.no)

These authors contributed equally: Siri Leknes, Monica Sarfi.

Received: 17 January 2019 Revised: 12 March 2019 Accepted: 24 March 2019

Published online: 31 March 2019 
to assess potential differences in decision sub-processes. This approach allows for a fine-grained comparison of the mechanisms underpinning reward-based decisions between groups. The DDM yields estimates of key decision parameters: speed-accuracy tradeoff, time spent on perceptual and motor processes, signal processing efficiency and stimulus preference. We expected that potential group differences in cognitive processing would be reflected in one or several of these parameters. To establish the typical range of healthy response bias in the PRT, we performed a meta-analysis of performance of 968 healthy controls from previous studies.

In summary, we measured reward responsiveness in OMT using three distinct approaches (self-report, behavioral test, and decision sub-processes), and included two independent comparison samples (total $n=995$ ). Reductions in reward responsiveness in the OMT group would support the notion that chronic opioid agonist use, even in the relative absence of additional psychosocial vulnerability factors, can lead to enduring disruption of nondrug reward processing. In contrast, intact reward responsiveness in patients would provide evidence that long-term $\mu$-opioid receptor stimulation does not necessarily cause anhedonia.

\section{MATERIALS AND METHODS}

Participants

Fifty-six mothers were recruited as part of a 7-year follow-up of a longitudinal study of mothers in OMT (and their children). Three OMT mothers had recently discontinued drug treatment program (tapered), therefore the final sample included 23 mothers in current OMT (in treatment for $>7$ years) and 30 comparison mothers (Table 1). Apart from six foster mothers in the comparison group, participants were recruited during pregnancy in 2005-2007; group characteristics at study inclusion are described in [31]. Requirements for entering OMT include an ICD-10 opioid dependence diagnosis and a medical evaluation of treatment eligibility. Lifetime injection of heroin was on average 8 years before entering OMT [32].

Comparison mothers with no reported illicit drug use or psychiatric illness were matched (at the time of study inclusion) to the OMT group on age, gender, and time of pregnancy (apart from the six foster mothers) but not tobacco use or years of education. Therefore we refer to a 'comparison' (COMP) rather than 'control group'.

Procedure

Procedures were approved by the Regional Ethics Committee (2013/1606/REK Sør-Øst B). Participants received verbal and written information about the study and signed a separate consent form before completing the reward responsiveness tasks. Participants received 10-15 USD corresponding to task performance.

\section{Measures}

Characterizing the study groups. We used the 25-item Shortened Hopkins Symptom Checklist [SCL-25, [33]] and a cut-off of 1.0 to identify participants with at least some anxiety and/or depression [34]. General life-satisfaction was operationalized as the mean rating [35] of the life-satisfaction questionnaire [LISAT-9, [36]]. We also employed the behavioral approach/inhibition scale [BIS/BAS, [37]]. A locally developed questionnaire based on [38, 39] was used to assess current mood and potential opiate side effects on an electronic visual analogue scale (VAS).

Subjective reward responsiveness. The state measure of anhedonia was modified [40] from the Snaith-Hamilton Pleasure Scale [SHAPS, [41]]. Unlike the original version, which centers on hypothetical enjoyment of everyday rewards (e.g., food, social contact, and esthetics) during the last few days, the present version assessed current hedonic state ("Right now I would [appreciate/enjoy/etc]") as a means to avoid recall bias [42]. Further, to capture variance in hedonic capacity in both patients and healthy participants, items were rated on an electronic VAS ("not at all"-_extremely").

Objective reward responsiveness. Behavioral reward responsiveness was measured with the well-established PRT, a perceptual decision task with skewed rewards [sometimes called the 'objective anhedonia test'; $[43,44]]$. In the majority of healthy participants, the skewed reward schedule induces a response bias reflecting the propensity to change behavior as a function of reward. During each trial, participants see a mouthless schematic face (Fig. 1) for $500 \mathrm{~ms}$ before the mouth is briefly presented (100 $\mathrm{ms})$. The task is to identify which of two possible mouths was presented (long/short). The marginal difference in mouth length (11 and $12 \mathrm{~mm}$ ) together with the brief presentation time makes the identification challenging (piloted to yield an average accuracy of $75 \%)$. Participants are instructed that upon correct identification of the mouth there is a chance to receive a small monetary reward (NOK 1, 12 cents). For one stimulus, $75 \%$ of all correct identifications are rewarded (termed the rich response option) and for the other stimulus only $25 \%$ of the correctly identified mouths lead to reward (lean option). This reward schedule is unknown to participants, who nevertheless develop a response bias towards the rich stimulus. Participants rarely become aware of this reward skewed schedule [e.g., see [45-47]]. The experiment consisted of three blocks of 100 trials separated by short breaks (self-paced). The task was implemented using E-Prime $2.0^{\circ}$ (Psychology Software Tools Inc., Pittsburg, PA, USA).

\section{Statistical analyses}

Experimental data were analyzed using $\mathrm{R}$ [version 3.1.2, [48]] and IBM SPSS (version 24). Group differences on questionnaire measures were assessed using independent samples $t$-tests (Welch's $t$-test when Levene's test indicated unequal variances). Mixed effects ANOVAs were used for experimental task data. Greenhouse-Geisser correction was employed when sphericity assumptions were violated. For non-significant group contrasts that are interpreted in the discussion section, Bayes Factor, which gives information about the relative evidence for two competing models $\left(\mathrm{H}_{1}\right.$ : group difference vs $\mathrm{H}_{0}$ : no group difference) given the data, was calculated in $\mathrm{R}$ using the BayesFactor package [49].

Missing data and outlier exclusion. The final number of participants included in the behavioral task analysis was OMT $=20$ and $\mathrm{COMP}=27$ (one dataset from each group was lost; two OMT and one COMP participants were excluded due to failure to follow task instructions; one COMP failed to complete the task). Trials with responses $<250 \mathrm{~ms}$ and $>2500 \mathrm{~ms}$ were excluded prior to analysis (97 trials, $0.7 \%$ ). Some responses to individual questionnaire items were also missing (see Table 1). Tukey's method was used to identify extreme outliers ranged above and below the $1.5^{*} \mathrm{IQR}$ (inter quartile range), resulting in removal of one OMT data point from the subjective reward responsiveness dataset prior to analysis.

Experimental task data. Reaction time and accuracy (precision) data from the PRT were analyzed using ANOVA with block (1, 2, and 3) and stimulus type (rich, lean) as within-subjects factors and group (OMT, COMP) as between-subjects factor. In addition to accuracy and reaction time measures, we calculated response bias $(\log b)$ and discriminability $\left(d^{\prime}\right)$ in concordance with established procedures for signal detection analysis [50]. 0.5 was added to 
Table 1. Group characteristics and questionnaire measures

\begin{tabular}{|c|c|c|c|c|c|c|c|}
\hline & \multicolumn{3}{|c|}{$\begin{array}{l}\text { Opioid maintenance treatment } \\
\text { group }\end{array}$} & \multicolumn{2}{|c|}{ Comparison group } & \multirow[b]{2}{*}{$t(d f)$} & \multirow[b]{2}{*}{$p$} \\
\hline & Mean & SD & Range & Mean & SD & & \\
\hline Education in years & 10.3 & 1.8 & & 15.34 & 3.50 & $6.7(43.4)$ & $<0.001 \#$ \\
\hline \multicolumn{8}{|l|}{ Medication assisted treatment } \\
\hline Methadone $(n=15)$ : years in treatment & 10.7 & 2.3 & & NA & NA & & \\
\hline Dose, median mg per day ${ }^{\mathrm{a}}$ & 100 & 115.9 & $5-440$ & NA & NA & & \\
\hline \multicolumn{8}{|l|}{ Smoking last year } \\
\hline None $(n)$ & 3 & & & 16 & & & \\
\hline$\leq 5$ cigarettes a day $(n)$ & 3 & & & 13 & & & \\
\hline Never $(n)$ & 5 & & & 2 & & & \\
\hline$<5$ times a year $(n)$ & 11 & & & 18 & & & \\
\hline $2-3$ times a month $(n)$ & 4 & & & 1 & & & \\
\hline $1-3$ times a week $(n)$ & 2 & & & 9 & & & \\
\hline \multicolumn{8}{|l|}{$\mathrm{SCL}-25$} \\
\hline Anxiety Subscale & 0.82 & 0.86 & & 0.23 & 0.26 & $3.13(24.2)$ & $0.01 \#$ \\
\hline Depression Subscale & 0.99 & 0.87 & & 0.29 & 0.36 & $3.55(26.9)$ & $0.005 \#$ \\
\hline LISAT-9 & 3.76 & 0.97 & & 4.99 & 0.45 & $5.45(28.8)$ & $0.001 \#$ \\
\hline BIS & 2.76 & 0.58 & & 2.45 & 0.54 & $1.89(47)$ & 0.07 \\
\hline BAS overall score & 2.67 & 0.44 & & 2.46 & 0.46 & $1.62(47)$ & 0.11 \\
\hline
\end{tabular}

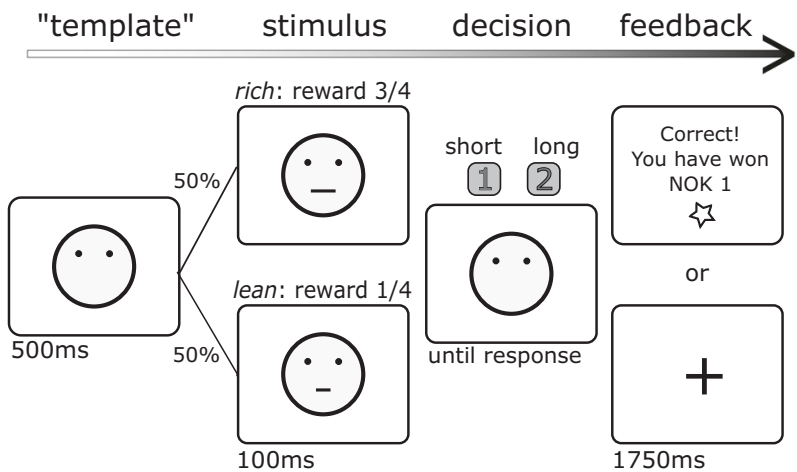

Fig. 1 Experimental task. Participants were presented with schematic faces and instructed to identify which of two alternative mouths was shown (short or long mouth). Unknown to the participant, correct identification of one of the alternatives lead to a monetary reward three times more often than the other alternative stimulus $(75 \%$ vs. $25 \%$ reward probability, rich and lean stimulus respectively). Non-rewarded and incorrect trials were followed by a fixation cross. The differences between the face stimuli have been inflated for illustrative purposes each cell when calculating $\log b$ and $\log d$.

$\log b=\frac{1}{2} \log \left(\frac{\text { rich }_{\text {correct }} \times \text { lean }_{\text {incorrect }}}{\text { rich }_{\text {incorrect }} \times \text { lean }_{\text {correct }}}\right)$

The log $b$ formula gives a log transformed ratio of presses to each of the two buttons associated with high or low probability of reward. This is an index of the reward sensitivity.

$\log d=\frac{1}{2} \log \left(\frac{\text { rich }_{\text {correct }} \times \text { lean }_{\text {correct }}}{\text { rich }_{\text {incorrect }} \times \text { lean }_{\text {incorrect }}}\right)$

The log $d$ formula gives a log transformed ratio of correct and incorrect responses. The discriminability indicates ability to distinguish between the two stimuli. For both measures we used mixed ANOVAs with block as within-subject and group as between-subject factor.

Meta-analysis: what is "normal" reward responsiveness?. To obtain a robust estimate of what constitutes a healthy range of response bias, we conducted a meta-analysis of previously published data on healthy participants' performance on the PRT. The metaanalysis included publications that have used the PRT and at least 
one group of healthy participants (see SM for the search and selection procedures). Data from 14 studies including 968 healthy (control group) participants was included and analyzed with a random-effects meta-analysis using the "metafor" package [51] in R statistical software [52] (Fig. 3, see supplementary materials (SM) for details).

DDM: group differences in processing of reward information?. To assess whether reward-based choices are processed differently in the two groups, accuracy and reaction times were fitted with a Bayesian hierarchical 4-parameter implementation of the DDM [[53]; see SM for details].

The DDM class of computational models is increasingly used in psychology and neuroscience, for instance to demonstrate how decision processes are altered by alcohol intoxication [nondecision time and drift rate; $[54,55]]$ and different psychiatric disorders [decision threshold and drift rate; [56, 57]]. It is a sequential sampling model that allows decomposition of reaction time and accuracy from two-alternative decisions into subcomponents reflecting mechanisms underpinning the observed effects of the task [58]. The DDM extends the classical signal detection analysis of two-alternative forced-choice data, in that it (i) includes trial-by-trial data instead of aggregated data and (ii) incorporates both reaction times and accuracy information to estimate characteristics of the decision process. Specifically, the DDM estimates parameters reflecting the efficiency of evidence accumulation (drift rate); decision caution (speed-accuracy tradeoff); potential a priori preference for either stimulus (starting point) and non-decision time (encoding and motor response) [59] (see SM Fig. S1).

\section{RESULTS}

\section{Group characteristics}

Group characteristics are presented in Table 1. Inspection of individual scores on the SCL-25 showed higher incidence of scores above the cut-off ( 1$)$ in the OMT group on both anxiety $\left(N_{\mathrm{OMT}}=8\right.$, $\left.N_{\text {COMP }}=1\right)$ and depression $\left(N_{\mathrm{OMT}}=8, N_{\text {COMP }}=2\right)$. As a group, mothers in OMT also showed significantly higher anxiety and depression symptoms, lower life satisfaction ( 1 point lower across life domains), and more discomfort in muscles and joints (Table S2 in SM), but no other significant group differences were observed on mood or state items (see SM), or on trait measures (BIS/BAS). Patients who remained in OMT after seven years did not differ in anxiety or depression scores at study inclusion (third trimester) from those who dropped out, nor did longitudinal analysis reveal substantial changes in anxiety and depression from inclusion until 7-year-follow up (see SM).

\section{Subjective reward responsiveness}

Self-report of state hedonic capacity was high in both the OMT and comparison group $\left(\mathrm{MEAN}_{\mathrm{OMT}}=6.59, \operatorname{MEAN}_{\mathrm{COMP}}=6.67\right.$, Welch's $t_{39.6}=0.201, p=0.844$ (95\% Cl: $-0.85,0.69$ ), see Fig. $2 \mathrm{a}$ ). Calculation of Bayes Factor $\left(\mathrm{BF}_{01}\right)$ indicated that the data are 3.42 times more likely given no group differences $\left(\mathrm{H}_{0}\right)$, than under the alternative hypothesis of different group means $\left(\mathrm{H}_{1} ; \mathrm{BF}_{10}\right.$ of 0.292 $\pm 0.02 \%)$. The OMT group's hedonic capacity ratings were comparable to scores from the 49 healthy men reported in [40]. For reasons of statistical power, methadone and buprenorphine users were not compared, however as illustrated in Fig. 2a there was no indication of systematic treatment differences.

Objective reward responsiveness task

Statistical analyses showed no significant main or interaction effects of group on accuracy, reaction time or discriminability (see SM for descriptive and inferential statistics).

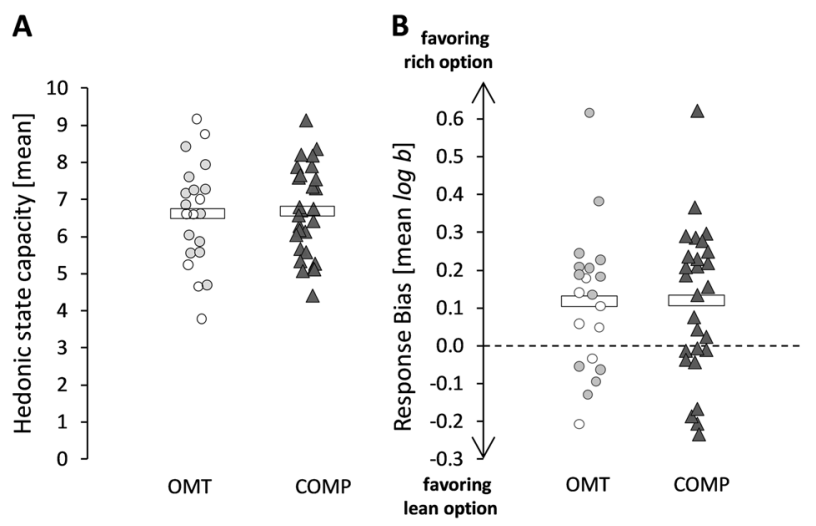

1459

Fig. 2 Subjective and objective reward responses. Individual data points are marked with circles (OMT) and triangles (COMP). The color of the circles indicates type of OMT: methadone (light gray); buprenorphine (white). Group means are indicated with transparent horizontal bars. Block-wise means and standard errors are reported in SM. For reasons of statistical power methadone and buprenorphine users were not directly compared. a Subjective reward responsiveness. Average rating on a visual analogue version of the Snaith-Hamilton Pleasure scale from 0 to 10 . Methadone: $n=14$; buprenorphine: $n=8$; b Objective reward responsiveness. Methadone: $n=13$; buprenorphine: $n=7$

Response bias. Objective reward responsiveness, operationalized as task response bias ( $\log b$ ) toward the rich stimulus, was evident in both groups (MEAN ${ }_{\text {OMT: }} 0.12$ (95\% Cl: 0.03-0.20); MEAN ${ }_{\text {СОMP: }}$ 0.12 (95\% Cl: $0.04-0.19)$, as confirmed by one-sample $t$-tests showing that both groups displayed reward responsiveness significantly different from zero (COMP: $t_{26}=3.07, p=0.005$, $\mathrm{BF}_{10}=8.6$, Cohen's $d=0.59$; OMT: $t_{19}=2.72, p=0.013, \mathrm{BF}_{10}=$ $3.98 ; d=0.61$ ). There was no group difference in bias (means were identical; $F_{1}, 45=0.002, p=0.97, p \eta^{2}<0.001$, see Fig. 2b). Response bias did not differ throughout the test (effect of block $\left.F_{1.52,} 68.5=0.817, p=0.46, p \eta^{2}=0.018\right)$ and there was no significant block ${ }^{*}$ group interaction $\left(F_{2,90}=0.048, p=0.95, p \eta^{2}=\right.$ $0.001)$. During informal debriefing after the session, none of the participants reported noticing the asymmetric reward schedule in this task. Bayes Factor $\left(\mathrm{BF}_{10}\right)$ for the group contrast was 0.183 , indicating that the data are 5.46 times more likely given no group differences $\left(\mathrm{H}_{0}\right)$, than under the alternative hypothesis of different group means.

'Healthy' response bias. The meta-analysis $(n=968)$ provided further confirmation that the OMT group displayed intact ('healthy') response bias in the objective reward responsiveness task (Fig. 3). The analysis yielded a mean response bias of 0.145 across the 14 studies included $(95 \% \mathrm{Cl}(0.13,0.16) ; p<0.001 ; \mathrm{SE}=$ 0.001 ). The sample of studies showed moderate heterogeneity ( $\tau 2$ $\left.<0.01 ; Q(d f=14)=24.57 ; p<0.03 ; I^{2}=47.1 \%\right)$.

Objective reward responsiveness task: Sub-processes. The computational modelling results corroborate the signal detection analysis of reward bias, showing the expected skewed starting points $(z)$ indicating a robust bias for the rich option for both the patients in OMT and the comparison group and individuals (Fig. 4b). Similarly, the efficiency of data accumulation for highreward probability trials (rich) was higher (mean (highest density interval, HDI): $1.5(1.3-1.7)$ ) than for the lean option (Mean: 1.16 (HDI: 0.8-1.5)). There was no evidence to support group differences on any of the examined subprocesses (Fig. 4a-d). See SM for details. 


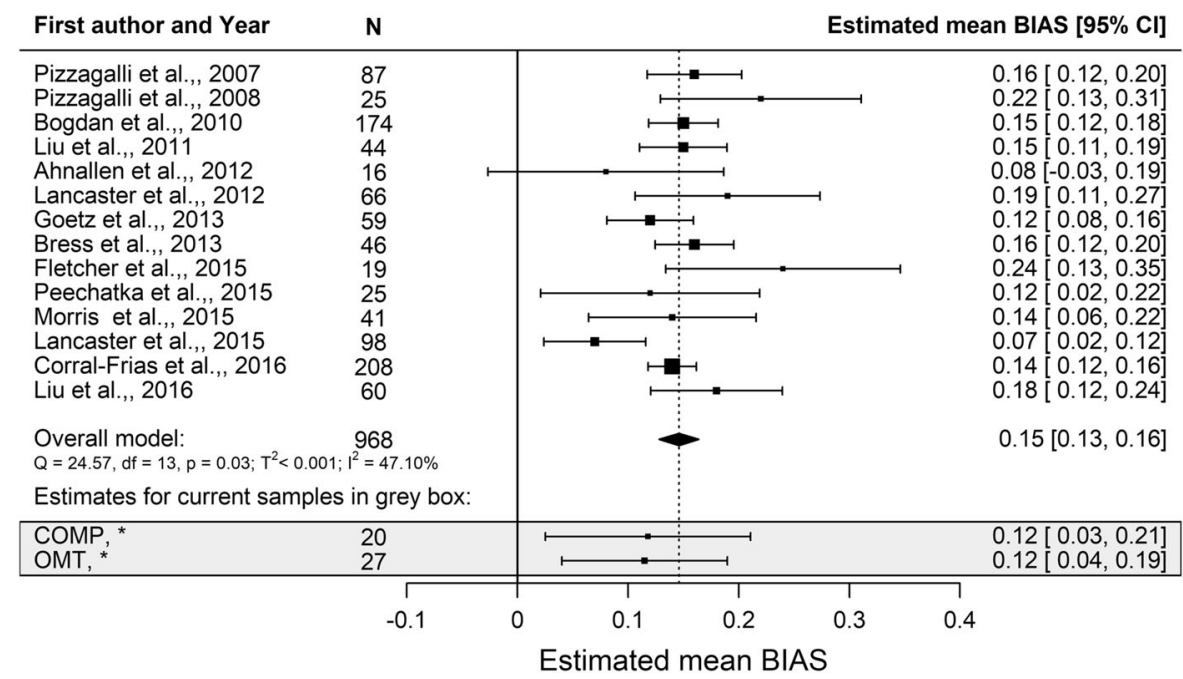

Fig. 3 Forest plot for meta-analysis of response bias (log b) across healthy groups of participants. $N=$ number of participants. Estimates from the two groups from the current study are shown below the meta-analytic results on the same $x$-axis in a gray box. Estimates are shown with two decimal precision as the majority of publications provide this level of detail. The zero-point indicates no bias (not changing behavior as a function of rewards received)
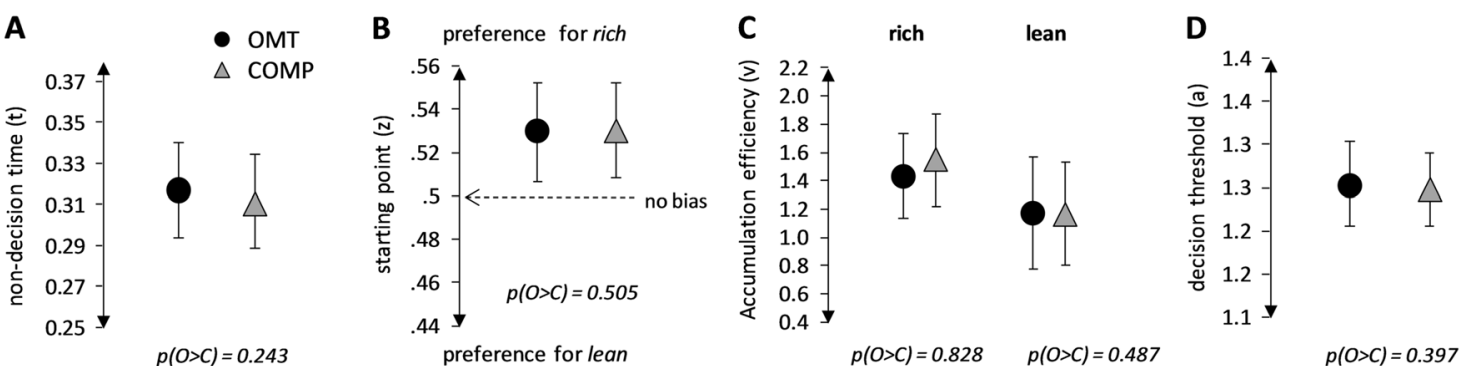

Fig. 4 Group estimates for drift diffusion model decision parameters. All panels indicate parameter means and 95\% HDI (highest density interval) from posterior distributions. Circles =OMT, triangles =COMP. a non-decision time; $\mathbf{b}$ starting point: 0.5 indicates no bias, higher numbers indicate preference for the high-reward-probability option (rich). c Evidence accumulation efficiency (drift rate) for rich and lean trials. d Decision threshold that indicates the speed-accuracy trade-off. Posterior probability for the group contrast is shown in italics $(\mathrm{O}=$ opioid maintenance group, $C=$ comparison group). Group contrasts $<0.05$ and $>0.95$ would indicate credible group differences

\section{DISCUSSION}

Across the measures and levels of analysis employed here, former heroin-addicted individuals in stable OMT displayed intact responsiveness to non-drug rewards. Importantly, the OMT group significantly and robustly modulated their behavior as a function of monetary reward probability. The degree of response bias towards the most frequently rewarded option was comparable with data from $\sim 1000$ healthy participants. Computational modelling revealed that despite previous heroin addiction and a minimum of 7 years of OMT, OMT patients' performance in this probabilistic reward task was comparable to a healthy sample even at the level of decision subprocesses. A self-report anhedonia measure also revealed high responsiveness to non-drug rewards in this unique OMT group. All patients were mothers who retained custody of their children after $>7$ years of OMT treatment. Accordingly, the OMT group tested here is characterized by fewer psychosocial vulnerability factors than other OMT cohorts [60, 61], but is also relatively small ( $N=20-23$ in key analyses).

The objective reward responsiveness task employed here measures participants' propensity to favor a more frequently rewarded response option. The task is designed to induce a bias that remains outside of conscious awareness. Disrupted response bias has been reported for several patient groups with subjective anhedonia [26, 50, 62-64], notably cannabis dependence [28], and nicotine withdrawal [29]. Since correct responses are rewarded with money in this task, the robust response bias demonstrated by the OMT group indicates intact sensitivity to a non-drug reward. Indeed, comparison with both a local comparison group and data from 968 healthy participants tested previously, confirmed that the patients' mean response bias fell within the normal range. This evidence for intact reward responsiveness after prolonged OMT contrasts with the notion that chronic opioid use causes anhedonia. For instance, OMT patients have shown blunted neural responses to non-drug reward [65-67]. However, most previous demonstrations of anhedonia in substance abuse have included patients during withdrawal or early abstinence [e.g., see reviews: [11, 12]]. In contrast, the OMT group tested here had been in stable treatment for an average of 9 years. A recent study pointed to illicit opiate use as a potential cause of self-reported anhedonia among patients in OMT [15]. In line with this evidence, illicit drug use is very limited in the OMT group tested here. To our knowledge, this is the first demonstration of intact ability to modify behavior to optimize reward in long-term OMT.

Somewhat surprisingly, computational modeling revealed that all the estimated decision subprocesses were comparable between study groups. Thus, we found no evidence for impairment in efficiency of information processing, response 
caution, degree of prior response preference, or non-decision time (time spent on perceptual and motor processes) in the OMT group. This result contrasts with previous reports that prolonged opioid use is associated with deficits in cognition and executive function, including decision making, cognitive flexibility, and memory [reviewed in [68]]. In active substance use disorder, immediate rewards are often favored impulsively [delayed discounting, [69, 70]]. Deficits in risky decision making [71-73] and prolonged reaction times $[66,74]$ have also been observed in some OMT groups. Here however, patients in OMT responded to stimuli with the same speed as comparison mothers. The lack of impairment in these underlying sensory, motor and cognitive processes corroborate the main results, i.e., that these patients were able to respond normally to non-drug rewards. Darke et al. [75] suggested that cognitive impairment may stem from indirect effects of opiates, such as lifestyle, poor health and nutrition, or exposure to violence. Notably, these vulnerability factors were less pronounced in the OMT patients tested here.

Acute stress induction [76] and high levels of daily stress [77] reduced reward responsiveness as measured by the probabilistic task used here. OMT using drugs with long-acting pharmacological properties (methadone and buprenorphine [78]) is thought to reduce stress [79] and supports normalized brain metabolite profiles, sex and stress hormone function [80-82]. Further, abnormal neural responses to drug cues in short-term OMT ( $<1$ year) were attenuated in patients with $2-3$ years of treatment [83]. In light of this literature, the present findings are consistent with the notion that long-term stable OMT could "renormalize" brain reward systems. An alternative explanation is that this sample's reward responsiveness was never impaired, potentially contributing to their successful treatment. Available longitudinal data from this cohort shows neither an improvement nor decline in symptoms of depression or anxiety. Further, we found no evidence of systematic differences in depression or anxiety levels at study inclusion between patients who did or did not remain in treatment (see SM for details). Future studies should characterize subjective or objective reward responsiveness in ongoing opioid addiction, and use longitudinal designs to test whether the measures could represent useful predictors of OMT treatment success [as suggested by [7]]. While the subgroups in our OMT cohort were too small to warrant statistical comparison, potential differential effects of methadone and buprenorphine on reward responsiveness should be addressed in future studies with larger patient groups.

We used a modified version of the SHAPS to assess subjective hedonic capacity in long-term OMT and healthy controls. The original questionnaire has been used in several previous studies of opioid dependence and misuse [15, 16, 84-86], revealing primarily large effect sizes (median Hedge's $g$ of $0.81, n>300$ opioid misusers in the references listed above). Here, the OMT and comparison group mean scores showed a negligible difference (0.08 on an 11-point scale). The objective reward responsiveness task employed here has also revealed impairments in substance dependent populations [28, 29]. An advantage to the present approach is that the ubiquitous nature of monetary reinforcement and the wide scope of pleasurable everyday scenarios included here render it unlikely that clinical anhedonia would go undetected in this population. Nonetheless, we observed somewhat higher scores of depression and anxiety in the OMT mothers. It is well established that not all depression involves anhedonia, and vice versa [87]. Note that we cannot exclude the possibility of a medium or small effect of OMT on non-drug reward responsiveness that cannot be detected here due to the small number of participants.

Several limitations of this study warrant consideration. The comparison group was matched to OMT patients on gender and age. Matching groups on potentially important variables such as smoking, socioeconomic status and years of education was not feasible. Nevertheless, despite somewhat higher self-reported distress in the OMT group, the two groups displayed strikingly similar performance on both the objective and subjective reward responsiveness measures. Importantly, performance was also comparable to data from a large group of healthy adults reported in the literature. We cannot exclude that other measures of responsiveness to rewards could reveal differences undetected here. Only women were tested in this study. The prevalence of opioid dependence differs in men and women [88] and most studies have tested mainly $[14,89]$ or exclusively men $[66,84,90]$. The relatively small number of patients included here limits the generalizability of results. Nevertheless, evidence of intact reward responsiveness in this group shows that normal reward behavior is possible even after a minimum of seven years in OMT preceded by on average eight years of heroin addiction.

The OMT group tested here is unique in a national and international context. Each patient was recruited during pregnancy and at the time of testing had received OMT for at least seven years. To maintain custody of their children, mothers in OMT in Norway commit to frequent testing and evaluation and are required to abstain from on-top illicit drug use. Compared to many other former heroin addicts, this subgroup leads a stable life-style with fewer psychosocial vulnerability factors. Furthermore, raising a child prompts the expression of caring behaviors, prioritizing others above oneself, and frequent problem solving. It is possible that the joys and responsibilities associated with child rearing may buffer against anhedonia in mothers in OMT. In support of this, a study of 390 Austrian mothers in OMT showed remarkably high self-reported quality of life [91]. Future studies should address the impact of psychosocial factors on reward responsiveness in current and former substance use disorder populations.

In sum, our findings suggest that long-term stable opioid agonist drug treatment does not necessarily lead to anhedonia or reduced reward responsiveness. The ability to adapt behavior according to non-drug reward may indicate a recovery of reward systems in long-term stable pharmacotherapy. Whether reward responsiveness is intact in OMT groups with more psychosocial difficulties remains to be seen. The present results may however extrapolate to other groups in long-term opioid treatment with a comparably small burden of psychosocial problems, such as patients with chronic pain. These results provide a new line of evidence supporting the utility of long-term opioid drug treatment for certain vulnerable patient groups.

\section{FUNDING AND DISCLOSURE}

The study was funded by a PhD grant from the South-Eastern Norway Regional Health Authority (2013053) and internal funding from The Norwegian Centre for Addiction Research (SERAF) to MS. $M E, P P L, M L P, N K, A M M, S L$, and MS reported no biomedical financial interests or potential conflicts of interest.

\section{ACKNOWLEDGEMENTS}

We are grateful to the many authors who provided information and data for the meta-analysis.

\section{ADDITIONAL INFORMATION}

Supplementary Information accompanies this paper at (https://doi.org/10.1038/ s41386-019-0377-9).

Publisher's note: Springer Nature remains neutral with regard to jurisdictional claims in published maps and institutional affiliations.

\section{REFERENCES}

1. Koob GF, Volkow ND. Neurobiology of addiction: a neurocircuitry analysis. Lancet Psychiatry.. 2016;3:760-73. 
2. Elman I, Borsook D. Common brain mechanisms of chronic pain and addiction. Neuron. 2016;89:11-36.

3. Nestler EJ. Is there a common molecular pathway for addiction? Nat. Neurosci. 2005:8:1445-49.

4. Robinson TE, Berridge KC. The incentive sensitization theory of addiction: Some current issues. Philos Trans R Soc B. 2008;363:3137-46.

5. Robinson TE, Berridge KC. The neural basis of drug craving: an incentivesensitization theory of addiction. Brain research reviews. 1993;18:247-91.

6. Volkow ND, Wang G-J, Fowler JS, Tomasi D, Telang F, Baler R. Addiction: Decreased reward sensitivity and increased expectation sensitivity conspire to overwhelm the brain's control circuit. Bioessays. 2010;32:748-55.

7. Lubman DI, Yücel M, Kettle JWL, Scaffidi A, MacKenzie T, Simmons JG, et al. Responsiveness to drug cues and natural rewards in opiate addiction: associations with later heroin use. Arch Gen Psychiatry. 2009;66:205-13.

8. Lubman DI, Peters LA, Mogg K, Bradley BP, Deakin JFW. Attentional bias for drug cues in opiate dependence. Psychol Med. 2000;30:169-75.

9. Zijlstra F, Veltman DJ, Booij J, van den Brink W, Franken IHA. Neurobiological substrates of cue-elicited craving and anhedonia in recently abstinent opioiddependent males. Drug Alcohol Depend. 2009;99:183-92.

10. Garland EL, Froeliger B, Howard MO. Allostatic dysregulation of natural reward processing in prescription opioid misuse: autonomic and attentional evidence. Biol Psychol. 2015;105:124-29.

11. Hatzigiakoumis DS, Martinotti G, Di Giannantonio M, Janiri L, Hatzigiakoumis D, Martinotti G, et al. Anhedonia and substance dependence: clinical correlates and treatment options. Frontiers Psychiatry. 2011;2:10.

12. Garfield JBB, Lubman DI, Yücel M. Anhedonia in substance use disorders: A systematic review of its nature, course and clinical correlates. Aust N Z J Psychiatry. 2014;48:36-51.

13. Treadway MT, Zald DH. Reconsidering anhedonia in depression: Lessons from translational neuroscience. Neurosci Biobehav Rev. 2011;35:537-55.

14. Janiri L, Martinotti G, Dario T, Reina D, Paparello F, Pozzi G, et al. Anhedonia and substance-related symptoms in detoxified substance-dependent subjects: a correlation study. Neuropsychobiology. 2005;52:37-44.

15. Garfield JBB, Cotton SM, Allen NB, Cheetham A, Kras M, Yücel M, et al. Evidence that anhedonia is a symptom of opioid dependence associated with recent use. Drug Alcohol Depend. 2017;177:29-38.

16. Huhn AS, Meyer RE, Harris JD, Ayaz H, Deneke E, Stankoski DM, et al. Evidence of anhedonia and differential reward processing in prefrontal cortex among postwithdrawal patients with prescription opiate dependence. Brain Res Bull. 2016;123:102-09.

17. Karow A, Reimer J, Schäfer I, Krausz M, Haasen C, Verthein U. Quality of life under maintenance treatment with heroin versus methadone in patients with opioid dependence. Drug Alcohol Depend. 2010;112:209-15.

18. Koob GF, Arends MA, Le Moal M. Drugs, addiction, and the brain. Academic Press; San Diego, 2014.

19. Whiteford HA, Degenhardt L, Rehm J, Baxter AJ, Ferrari AJ, Erskine HE, et al. Global burden of disease attributable to mental and substance use disorders: findings from the Global Burden of Disease Study. Lancet. 2010;382:1575-86.

20. Mattick RP, Breen C, Kimber J, Davoli M. Buprenorphine maintenance versus placebo or methadone maintenance for opioid dependence. Cochrane Database Syst Rev. 2014, CD002207. https://doi.org/10.1002/14651858.CD002207.pub4.

21. World.Health.Organization. Treatment of opioid dependence. 2012. http://www. who.int/substance_abuse/activities/treatment_opioid_dependence/en/index. html.

22. Bukten A, Skurtveit S, Gossop M, Waal H, Stangeland P, Havnes I, et al. Engagement with opioid maintenance treatment and reductions in crime: a longitudinal national cohort study. Addiction. 2012;107:393-99.

23. Amato L, Davoli M, Perucci CA, Ferri M, Faggiano F, Mattick RP. An overview of systematic reviews of the effectiveness of opiate maintenance therapies: available evidence to inform clinical practice and research. J Subst Abuse Treat. 2005;28:321-29.

24. Donoghoe MC, Dolan KA, Stimson GV. Life-style factors and social circumstances of syringe sharing in injecting drug users. Br J Addict. 1992;87:993-1003.

25. Vrieze E, Demyttenaere K, Bruffaerts $R$, Hermans D, Pizzagalli DA, Sienaert $P$, et al. Dimensions in major depressive disorder and their relevance for treatment outcome. J Affect Disord. 2013;155:35-41.

26. Pechtel P, Dutra SJ, Goetz EL, Pizzagalli DA. Blunted reward responsiveness in remitted depression. J Psychiatr Res. 2013;47:1864-69.

27. Berghorst LH, Bogdan R, Frank MJ, Pizzagalli DA. Acute stress selectively reduces reward sensitivity. Front Hum Neurosci. 2013;7:133.

28. Lawn W, Freeman TP, Pope RA, Joye A, Harvey L, Hindocha C, et al. Acute and chronic effects of cannabinoids on effort-related decision-making and reward learning: an evaluation of the cannabis 'amotivational' hypotheses. Psychopharmacology. 2016;233:3537-52.
29. Pergadia ML, Der-Avakian A, D'Souza MS, et al. Association between nicotine withdrawal and reward responsiveness in humans and rats. JAMA Psychiatry. 2014;71:1238-45.

30. Ratcliff R, McKoon G. The Diffusion Decision Model: Theory and Data for TwoChoice Decision Tasks. Neural Comput. 2007;20:873-922.

31. Sarfi M, Smith $L$, Waal $H$, Sundet JM. Risks and realities: dyadic interaction between 6-month-old infants and their mothers in opioid maintenance treatment. Infant Behav Dev. 2011;34:578-89.

32. Lund IO, Skurtveit S, Sarfi M, Bakstad B, Welle-Strand G, Ravndal E. Substance use during and after pregnancy among a national cohort of pregnant women in opioid maintenance treatment and their partners. J Subst Use. 2012;17: 277-86.

33. Derogatis LR, Lipman RS, Rickels K, Uhlenhuth EH, Covi L. The Hopkins Symptom Checklist (HSCL): a self-report symptom inventory. Behav. Sci. 1974;19:1-15.

34. Lund IO, Skurtveit S, Sarfi M, Bakstad B, Welle-Strand G, Ravndal E. A 2-year prospective study of psychological distress among a national cohort of pregnant women in opioid maintenance treatment and their partners. J Subst Use. 2013;18:148-60.

35. Post MW, de Witte LP, van Asbeck FW, van Dijk AJ, Schrijvers AJ. Predictors of health status and life satisfaction in spinal cord injury. Arch Phys Med Rehabil. 1998;79:395-401.

36. Fugl-Meyer AR, Bränholm I-B, Fugl-Meyer KS. Happiness and domain-specific life satisfaction in adult northern Swedes. Clin Rehabil. 1991;5:25-33.

37. Carver CS, White TL. Behavioral Inhibition, Behavioral Activation, and Affective Responses to Impending Reward and Punishment: The BIS/BAS Scales. J Pers Soc Psychol. 1994;67:319-33.

38. Walker DJ, Zacny JP. Subjective, psychomotor, and analgesic effects of oral codeine and morphine in healthy volunteers. Psychopharmacology. 1998;140: 191-201.

39. Comer S, Cooper Z, Kowalczyk W, Sullivan M, Evans S, Bisaga A, et al. Evaluation of potential sex differences in the subjective and analgesic effects of morphine in normal, healthy volunteers. Psychopharmacology. 2010;208:45-55.

40. Eikemo M, Løseth GE, Johnstone T, Gjerstad J, Willoch F, Leknes S. Sweet taste pleasantness is modulated by morphine and naltrexone. Psychopharmacology (Berl). 2016:233:3711-23.

41. Snaith RP, Hamilton M, Morley S, Humayan A, Hargreaves D, Trigwell P. A scale for the assessment of hedonic tone. The Snaith-Hamilton Pleasure Scale. Br J Psychiatry. 1995;167:99-103.

42. Ready RE, Weinberger MI, Jones KM. How happy have you felt lately? Two diary studies of emotion recall in older and younger adults. Cognition Emotion. 2007;21:728-57.

43. Pizzagalli DA, Sherwood RJ, Henriques JB, Davidson RJ. Frontal brain asymmetry and reward responsiveness: a source-localization study. Psychol Sci. 2005;16: 805-13.

44. Tripp G, Alsop B. Sensitivity to reward frequency in boys with attention deficit hyperactivity disorder. J Clin Child Adolesc Psychol. 1999;28:366-75.

45. Eikemo M, Biele G, Willoch F, Thomsen L, Leknes S. Opioid modulation of valuebased decision making in healthy humans. Neuropsychopharmacology. 2017;42: 1833-40.

46. Heerey EA, Bell-Warren KR, Gold JM. Decision-making impairments in the context of intact reward sensitivity in Schizophrenia. Biol. Psychiatry. 2008;64:62-69.

47. Lancaster TM, Linden DE, Heerey EA. COMT val158met predicts reward responsiveness in humans. Genes, Brain Behav. 2012;11:986-92.

48. Team RC. (R Foundation for Statistical Computing, Vienna, Austria, 2014)

49. Morey RD, Rouder JN. BayesFactor: Computation of Bayes Factors for Common Designs. R package version 0.9.12-2. 2015. http://CRAN.R-project.org/ package $=$ BayesFactor.

50. Pizzagalli DA, losifescu D, Hallett LA, Ratner KG, Fava M. Reduced hedonic capacity in major depressive disorder: evidence from a probabilistic reward task. J Psychiatr Res. 2008;43:76-87.

51. Viechtbauer W. Conducting Meta-Analyses in R with the metafor Package. 2010. 2010;36:48.

52. R Core Team. (R Foundation for Statistical Computing, Vienna, Austria, 2016)

53. Wiecki TV, Sofer I, Frank MJ. HDDM: hierarchical bayesian estimation of the driftdiffusion model in python. Front Neuroinform. 2013;7:14.

54. Van Ravenzwaaij D, Dutilh G, Wagenmakers EJ. A diffusion model decomposition of the effects of alcohol on perceptual decision making. Psychopharmacology. 2012;219:1017-25

55. Stock A-K, Hoffmann S, Beste C. Effects of binge drinking and hangover on response selection sub-processes-a study using EEG and drift diffusion modeling. Addict Biol. 2016;22:1355-65.

56. Mulder MJ, Bos D, Weusten JMH, Van Belle J, Van Dijk SC, Simen P, et al. Basic impairments in regulating the speed-accuracy tradeoff predict symptoms of attention-deficit/hyperactivity disorder. Biol. Psychiatry. 2010;68:1114-19. 
57. Banca P, Vestergaard MD, Rankov V, Baek K, Mitchell S, Lapa T, et al. Evidence accumulation in obsessive-compulsive disorder: the role of uncertainty and monetary reward on perceptual decision-making thresholds. Neuropsychopharmacology. 2015;40:1192-202.

58. Ratcliff R, McKoon G. The diffusion decision model: theory and data for twochoice decision tasks. Neural Comput. 2008;20:873-922.

59. Voss A, Rothermund K, Voss J. Interpreting the parameters of the diffusion model: an empirical validation. Mem Cognit. 2004;32:1206-20.

60. Martin PR, Arria AM, Fischer G, Kaltenbach K, Heil SH, Stine SM, et al. Psychopharmacologic management of opioid-dependent women during pregnancy. Am J Addict. 2009;18:148-56.

61. Velez M, Jansson LM. The Opioid dependent mother and newborn dyad: nonpharmacologic care. J Addict Med. 2008;2:113-20.

62. Abramovitch A, Pizzagalli DA, Reuman L, Wilhelm S. Anhedonia in obsessivecompulsive disorder: beyond comorbid depression. Psychiatry Res. 2014;216: 223-9.

63. Fletcher K, Parker G, Paterson A, Fava M, losifescu D, Pizzagalli DA. Anhedonia in melancholic and non-melancholic depressive disorders. J Affect Disord. 2015; 184:81-88.

64. Pizzagalli DA, Jahn AL, O'Shea JP. Toward an objective characterization of an anhedonic phenotype: a signal-detection approach. Biol. Psychiatry. 2005;57: 319-27.

65. Martin-Soelch C, Chevalley AF, Kunig G, Missimer J, Magyar S, Mino A, et al. Changes in reward-induced brain activation in opiate addicts. Eur J Neurosci. 2001;14:1360-68.

66. Gradin VB, Baldacchino A, Balfour D, Matthews K, Steele JD. Abnormal brain activity during a reward and loss task in opiate-dependent patients receiving methadone maintenance therapy. Neuropsychopharmacology. 2014;39:885-94.

67. Asensio S, Romero MJ, Palau C, Sanchez A, Senabre I, Morales JL, et al. Altered neural response of the appetitive emotional system in cocaine addiction: an fMR Study. Addict Biol. 2010;15:504-16.

68. Prosser J, Cohen LJ, Steinfeld M, Eisenberg D, London ED, Galynker II. Neu ropsychological functioning in opiate-dependent subjects receiving and following methadone maintenance treatment. Drug Alcohol Depend. 2006;84:240-47.

69. Reynolds B. A review of delay-discounting research with humans: relations to drug use and gambling. Behav Pharmacol. 2006;17:651-67.

70. Madden GJ, Petry NM, Badger GJ, Bickel WK. Impulsive and self-control choices in opioid-dependent patients and non-drug-using control patients: Drug and monetary rewards. Exp Clin Psychopharmacol. 1997;5:256-62.

71. Petry NM, Bickel WK, Arnett M. Shortened time horizons and insensitivity to future consequences in heroin addicts. Addiction. 1998;93:729-38.

72. Ersche KD, Fletcher PC, Roiser JP, Fryer TD, London M, Robbins TW, et al. Differences in orbitofrontal activation during decision-making between methadonemaintained opiate users, heroin users and healthy volunteers. Psychopharmacology. 2006;188:364-73.

73. Pirastu R, Fais R, Messina M, Bini V, Spiga S, Falconieri D, et al. Impaired decisionmaking in opiate-dependent subjects: effect of pharmacological therapies. Drug Alcohol Depend. 2006;83:163-68.

74. Rogers RD, Everitt B, Baldacchino A, Blackshaw A, Swainson R, Wynne K, et al. Dissociable deficits in the decision-making cognition of chronic amphetamine abusers, opiate abusers, patients with focal damage to prefrontal cortex, and tryptophan-depleted normal volunteers: evidence for monoaminergic mechanisms. Neuropsychopharmacology. 1999;20:322-39.

75. Darke S, Sims J, McDonald S, Wickes W. Cognitive impairment among methadone maintenance patients. Addiction. 2000;95:687-95.

76. Bogdan R, Pizzagalli DA. Acute stress reduces reward responsiveness: implications for depression. Biol. Psychiatry. 2006;60:1147-54.

77. Pizzagalli DA, Bogdan R, Ratner KG, Jahn AL. Increased perceived stress is associated with blunted hedonic capacity: potential implications for depression research. Behav Res Ther. 2007;45:2742-53.

78. Kreek MJ. Methadone-related opioid agonist pharmacotherapy for heroin addiction: history, recent molecular and neurochemical research and future in mainstream medicine. Ann N Y Acad Sci. 2000;909:186-216.

79. Koob G, Kreek MJ. Stress, dysregulation of drug reward pathways, and the transition to drug dependence. Am J Psychiatry. 2007;164:1149-59.

80. Kaufman MJ, Pollack MH, Villafuerte RA, Kukes TJ, Rose SL, Mendelson JH, et al. Cerebral phosphorus metabolite abnormalities in opiate-dependent polydrug abusers in methadone maintenance. Psychiatry Re Neuroimaging. 1999;90: 143-52.

81. Kreek MJ. Medical complications in methadone patients. Ann N Y Acad Sci. 1978;311:110-34.

82. Kreek MJ, Wardlaw SL, Hartman N, Raghunath J, Friedman J, Schneider B, et al Circadian rhythms and levels of $\beta$-endorphin, acth, and cortisol during chronic methadone maintenance treatment in humans. Life Sci. 1983;33:409-11.

83. Wang Y, Wang H, Li W, Zhu J, Gold MS, Zhang D, et al. Reduced responses to heroin-cue-induced craving in the dorsal striatum: effects of long-term methadone maintenance treatment. Neurosci Lett. 2014;581:120-24.

84. Stevens A, Peschk I, Schwarz J. Implicit learning, executive function and hedonic activity in chronic polydrug abusers, currently abstinent polydrug abusers and controls. Addiction. 2007;102:937-46.

85. Franken $\mathrm{IH}$, Rassin $\mathrm{E}$, Muris $\mathrm{P}$. The assessment of anhedonia in clinical and nonclinical populations: further validation of the Snaith-Hamilton Pleasure Scale (SHAPS). J Affect Disord. 2007;99:83-89.

86. Meshesha LZ, Pickover AM, Teeters JB, Murphy JG. A longitudinal behavioral economic analysis of non-medical prescription opioid use among college students. Psychol Rec. 2017;67:241-51.

87. Leventhal AM, Chasson GS, Tapia E, Miller EK, Pettit JW. Measuring hedonic capacity in depression: a psychometric analysis of three anhedonia scales. J Clin Psychol. 2006;62:1545-58.

88. Greenfield SF, Back SE, Lawson K, Brady KT. Substance abuse in women. Psychiatr Clin North Am. 2010;33:339-55.

89. Zhang R, Jiang G, Tian J, Qiu Y, Wen X, Zalesky A, et al. Abnormal white matter structural networks characterize heroin-dependent individuals: a network analysis. Addict Biol. 2016;21:667-78.

90. Lubman DI, Allen NB, Peters LA, Deakin JFW. Electrophysiological evidence that drug cues have greater salience than other affective stimuli in opiate addiction. J Psychopharmacol. 2008;22:836-42.

91. Metz VE, Comer SD, Wuerzl J, Pribasnig A, Fischer G. Characteristics and quality of life of opioid-dependent pregnant women in Austria. Archives Women's Mental Health. 2014;17:529-39. 